\title{
A molecular spin-crossover film allows for wavelength tuning the resonance of a Fabry-Perot cavity
}

\author{
Yuteng Zhang, ${ }^{\mathrm{a}, \mathrm{b}}$ Karl Ridier, ${ }^{\mathrm{a}}$ Victoria Shalabaeva, ${ }^{\mathrm{a}}$ Isabelle Séguy, ${ }^{\mathrm{b}}$ Sylvain Pelloquin, ${ }^{\mathrm{b}}$ Henri \\ Camon, ${ }^{\mathrm{b}}$ Stéphane Calvez, ${ }^{\mathrm{b}}$ Lucie Routaboul, ${ }^{\mathrm{a}}$ Lionel Salmon, ${ }^{\mathrm{a}}$ Gábor Molnár, ${ }^{*}$, a Azzedine \\ Bousseksou ${ }^{*}$, a
}

\begin{abstract}
We harness the remarkable refractive index switching $(0.04<\Delta n(\lambda)<0.2)$ between the low-spin and high-spin states of the molecular spin-crossover (SCO) complex $\left[\mathrm{Fe}\left(\mathrm{HB}(1,2,4-\text { triazol-1-yl })_{3}\right)_{2}\right]$ to realize a wavelength-tunable $\mathrm{Ag} / \mathrm{SCO} / \mathrm{Ag}$ multilayer Fabry-Perot cavity. This tuneability is coupled with low absorption losses $\left(k \leq 10^{-4}\right)$ between $350-1000 \mathrm{~nm}$ providing scope for reconfigurable and self-adaptive photonics applications in the visible spectral region.
\end{abstract}

Materials that undergo electronic and/or structural phase change associated with a reversible switching of their optical properties have received recently increasing attention for the development of active photonic devices. ${ }^{1-2}$ These phase-change materials (PCMs) exhibit changes of their refractive index in response to external stimuli (e.g. heat, voltage bias or light irradiation), which have been exploited for a variety of applications, such as smart windows, optical memories, spatial light modulators and photonic integrated circuits. ${ }^{3-11}$ The most widely studied PCMs are chalcogenide alloys (e.g. $\mathrm{Ge}_{2} \mathrm{Sb}_{2} \mathrm{Te}_{5}$ ) showing amorphous-crystalline phase change, transition metal oxides (e.g. $\mathrm{VO}_{2}$ ) exhibiting metal-insulator transitions, and liquid crystals (e.g. 5CB) displaying order-disorder type transitions. Among these materials, however, mostly liquid crystals have been employed in practical applications requiring transparency in the visible spectral range. ${ }^{3}$ Yet, the intrinsic limitations of liquid crystals (slow speed, high scattering losses, intricate processing and need for encapsulation ${ }^{3}$ ) call for new material developments. ${ }^{4}$

In this context, emerging nanomaterials of molecular spin-crossover ( $\mathrm{SCO}$ ) compounds represent a promising novel class of PCMs with high potential for active photonics applications. ${ }^{12}$ These transition metal complexes (most often with $\mathrm{Fe}^{2+}$ metal centers) display reversible switching between their low-spin (LS) and high-spin (HS) electronic configurations under various external stimuli (temperature or pressure change, light or X-ray irradiation, etc.). ${ }^{13}$ The most apparent property of bulk SCO materials is the visible color change, which accompanies the switching event. On the other hand, much less recognized is the fact that the SCO is always associated with a substantial variation of the refractive index $(\Delta n=0.01-0.1)$ as well. This property arises due to combined electronic (polarizability change) and structural effects (unit cell volume change up to 15\%), albeit the latter contribution is assumed to prevail in most cases. ${ }^{14}$

In the past, the refractive index change in SCO materials has been investigated from the UV to the THz spectral ranges either as a means for detecting the spin transition in nanoscale objects or with the aim to develop switchable optical materials/devices. ${ }^{14-26}$ The latter comprise transient phase gratings, ${ }^{14}$ tunable Bragg filters for the sub-millimeter wavelength range, ${ }^{16}$ diffractive gas sensors $^{25}$ as well as active plasmonic switches based on lithographically patterned ${ }^{22}$ or, more recently, on chemically synthesized ${ }^{26}$ gold nano-rods. However, the SCO materials used in these works suffered from certain limitations, such as poor processability (e.g. powders), low environmental stability, weak cycling endurance and SCO far from room temperature. In the present Communication, we make use of the refractive index change associated with the SCO to render wavelength-tunable a Fabry-Perot resonator, using a high-quality SCO film, which effectively overcomes all these pitfalls, marking an important leap towards SCObased photonics.

Our work is based on thin films of the molecular SCO complex [Fe(HB(1,2,4-triazol-1-yl) $\left.\left.)_{3}\right)_{2}\right]$ (compound 1). High-quality, nanocrystalline thin films of 1 were recently developed using vacuum thermal evaporation, followed by water vapor annealing. ${ }^{27}$ These films exhibit a complete, well-defined and reproducible spin transition near $63-65^{\circ} \mathrm{C}$, depending only marginally on the film thickness ${ }^{28}$ and film defects ${ }^{29}$. Recent studies have demonstrated that the molecular spin state of these thin films can be modulated at high frequencies $\left(1-10 \mathrm{MHz}\right.$ ) with outstanding switching endurance (>107 cycles). ${ }^{30-31}$ Based on these attributes, films of 1 have been integrated into electronic/electromechanical test devices to explore their electrical resistance switching capabilities ${ }^{32-33}$ and their ability to induce mechanical actuation. ${ }^{34}$ and thermal damping ${ }^{35}$. In addition, they have been effectively used for nanoscale thermal imaging. ${ }^{31}$ 
In order to explore the photonic properties of 1, we have determined the optical constants $(n, k)$ of a nominally 100-nm-thick film (on top of a silicon substrate) using a Horiba UVISEL ellipsometer. Ellipsometric data were acquired for wavelengths between 250 and $1000 \mathrm{~nm}$ at various angles of incidence $\left(\mathrm{AOI}=56-60^{\circ}\right)$ and selected temperatures $\left(25-95^{\circ} \mathrm{C}\right)$, and fitted by a single oscillator model. ${ }^{36}$ Figures $1(\mathrm{a})$ and 1 (b) display the wavelength dependence of the refractive index $n$ and extinction coefficient $k$, respectively. Between 350 and $1000 \mathrm{~nm}, k$ remains below $c a .10^{-4}$ in the whole temperature range, whereas $n$ exhibits classical dispersion behavior. Indeed, in the Vis-NIR region, only a few Laporte-forbidden ligand-field transitions occur in 1 with absorption coefficients in the order of 100 and $10 \mathrm{~cm}^{-1}$ in the LS and HS states, respectively. These weak absorption bands are hardly detectable even in micrometer-thick films. ${ }^{30}$ Below $350 \mathrm{~nm}, k$ steeply increases and peaks at a value of 0.23 around $315 \mathrm{~nm}$, whereas $n$ exhibits
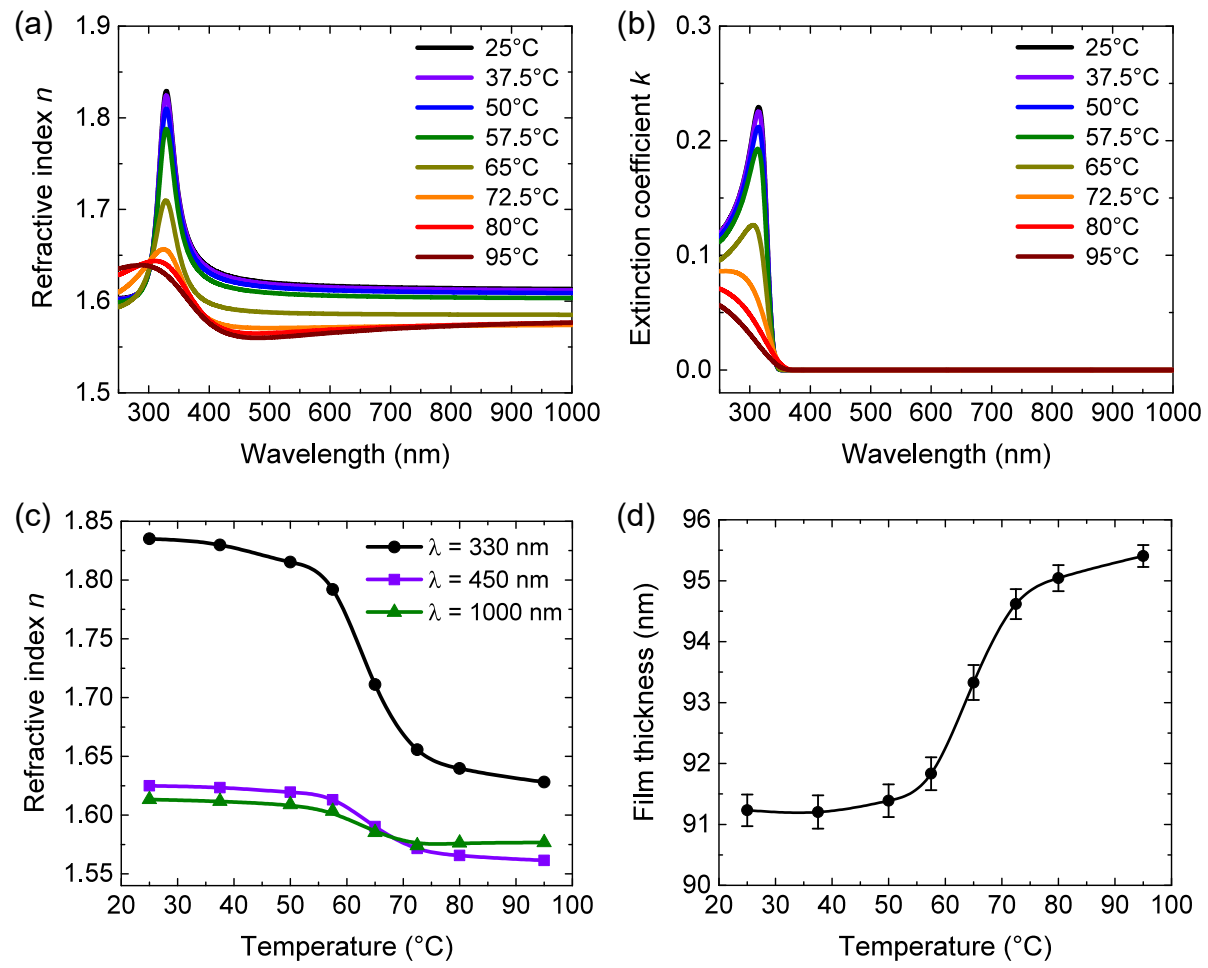

anomalous dispersion. The associated strong UV absorption is assigned to charge-transfer transitions in the LS state and it can be conveniently used to probe the SCO phenomenon in the thin films. ${ }^{27}$

Fig. 1. Ellipsometric characterization of a nominally 100-nm-thick film of 1. (a-b) Optical constants $(n, k)$ in the UV-Vis-NIR region (250-1000 nm) acquired at selected temperatures between 25 and $95{ }^{\circ} \mathrm{C}$. (c) Temperature dependence of the refractive index $n$ at selected wavelengths (heating mode). (d) Variation of the film thickness $t$ as a function of the temperature (heating mode).

The temperature dependences of the refractive index $n$ and film thickness $t$ are depicted in Figures 1 (c) and 1 (d), respectively. These curves show a compelling correlation. Far from the spin transition, both $n$ and $t$ are weakly temperature dependent, in agreement with the nearly zero thermal expansion coefficient of $1 .{ }^{37}$ Around the spin-transition temperature $\left(65{ }^{\circ} \mathrm{C}\right)$, the film thickness abruptly increases by $4.6 \%$, from $91.2(3)$ in the LS state to $95.4(2) \mathrm{nm}$ in the HS state. This variation is in reasonable agreement with the $5.6 \%$ expansion of the orthorhombic $c$-axis of the unit cell upon the SCO revealed by single crystal X-ray diffraction. ${ }^{37}$ (N.B. The film is oriented with the $c$-axis normal to the substrate. ${ }^{27}$ ) On the other hand, the value of $n$ concomitantly drops by ca. 0.2-0.04 (depending on the wavelength) at the LS-to-HS transition.

To translate this refractive index change into a functional property, we fabricated metal-based Fabry-Perot structures on glass substrates. Fabry-Perot interferometers are widely used in optical instruments, such as spectrometers, lasers, filters and so forth. ${ }^{38}$ They are constituted of a material (of arbitrary thickness) sandwiched in between two semi-transparent mirrors. Impinging light is bounced back and forth between the two mirrors and, at the resonance wavelength(s), effectively passes multiple times through the central material. As a result, the wavelength-selective constructive interaction with this layer occurs over an artificially increased length, which equivalently means that energy is stored in the device, which behaves as a resonator. The nominal design of our device, $\operatorname{Ag}(50 \mathrm{~nm}) / \mathrm{SCO}(115 \mathrm{~nm}) / \mathrm{Ag}(50 \mathrm{~nm})$, was set to exhibit a reflection (and a transmission) resonance in the green spectral range with a $Q$-factor (defined as the resonant wavelength divided by its full spectral width at half height) of 60 . Both the $\mathrm{Ag}$ and SCO layers were deposited by vacuum thermal evaporation ( $5 \times 10^{-7}$ Torr base pressure). To reach a high degree of crystallinity the as-deposited SCO film was solvent vapor annealed as described in ref. 27. A rotating, water-cooled substrate holder was used to achieve homogeneous and damage-free films. The normal-incidence transmittance and variable-incidence $(\mathrm{AOI}=8-$ $60^{\circ}$ ) reflectance characteristics of the devices were subsequently quantified, for both transverse electric (TE) and transverse 
magnetic (TM) polarizations, using a PerkinElmer Lambda 950 spectrophotometer. The temperature of the devices was controlled using a Peltier stage. Fortuitously, the transmittance window of the Ag films around $320 \mathrm{~nm}$ (associated with the silver plasma frequency) closely coincides with the LS absorption peak of $\mathbf{1}$. This allowed us to probe the optical absorbance change of $\mathbf{1}$ upon the spin transition - similar to neat films ${ }^{27}$. As shown in Figure 2(a), the spin transition in the cavity occurs in a reproducible and abrupt fashion near $65^{\circ} \mathrm{C}$. The total absorbance change $(\triangle O D=0.29)$ indicates that the spin transition is nearly complete in the 115-nm-thick film. ${ }^{28}$ These results unambiguously confirm that the presence of the Ag films - below and on top of the SCO layer does not substantially impact the spin-transition properties. (N.B. This observation was confirmed for the four investigated FabryPerot devices.) Figure 2 (b) shows representative reflectance spectra of the cavities recorded in the $\mathrm{LS}\left(25^{\circ} \mathrm{C}\right)$ and $\mathrm{HS}\left(80^{\circ} \mathrm{C}\right)$ states at various $\mathrm{AOI}$ under TE polarized illumination. As expected, the $\mathrm{Ag} / \mathrm{SCO} / \mathrm{Ag}$ multilayer structure exhibits pronounced resonances with quality factors $Q$ of $c a$. 30-35, i.e. approximately twice lower than predicted. Importantly, the resonance peaks display a blueshift (up to $8 \mathrm{~nm}$ ) when going from the LS to the HS state. This shift can be better appreciated in Figure 2(c), which shows a detailed temperature scan, as well as in Figure 2(d), which displays the dispersion of the cavity resonance in the LS and HS states for both TM and TE polarizations. The experimental behaviour can be fitted using conventional transfer matrix calculations ${ }^{38}$ including the known optical properties of silver ${ }^{39}$ and the above-reported ellipsometric data of the SCO film. The calculated characteristics are shown in Figures 2(b)-(d) in dotted lines. Taking into account a thickness expansion factor of the ScO film of $4.6 \%$ associated with the spin transition, a good agreement between the experimental and calculated angular dependences of the resonances is obtained for an effective (LS) SCO layer thickness of $119.0 \mathrm{~nm}$ and a silver thickness of $47.8 \mathrm{~nm}$, both of which being close to the targeted deposition values. However, to reach a better agreement between the experimental and simulated spectra (in particular concerning the resonance widths), it was necessary to introduce a surface roughness with a 1.7-nm-standard-deviation. This roughness parameter can be attributed to a combination of (glass) substrate roughness and evaporation-related contributions. Fig. 2. Optical properties of $\mathrm{Ag}(50 \mathrm{~nm}) / \mathrm{SCO}(115 \mathrm{~nm}) / \mathrm{Ag}(50 \mathrm{~nm})$ multilayer Fabry-Perot cavities. (a) Temperature dependence of the optical density change across the cavity $(\triangle O D=$ $\left.O D-O D_{H S}\right)$ at $\lambda=318 \mathrm{~nm}$ (normal incidence, non-polarized) along two heating-cooling cycles $\left(2^{\circ} \mathrm{C} \cdot \mathrm{min}^{-1} \mathrm{scan}\right.$ rate). (b) Reflectance spectra at selected AOI in the two spin states (TE polarization). (c) Cavity resonance (TE polarization, $\mathrm{AOI}=45^{\circ}$ ) as a function of temperature (heating mode). (d) Dispersion curves in the two spin states for TE and TM polarizations. The dotted lines in (b) and (d) were calculated by means of the transfer matrix method using ellipsometry data.
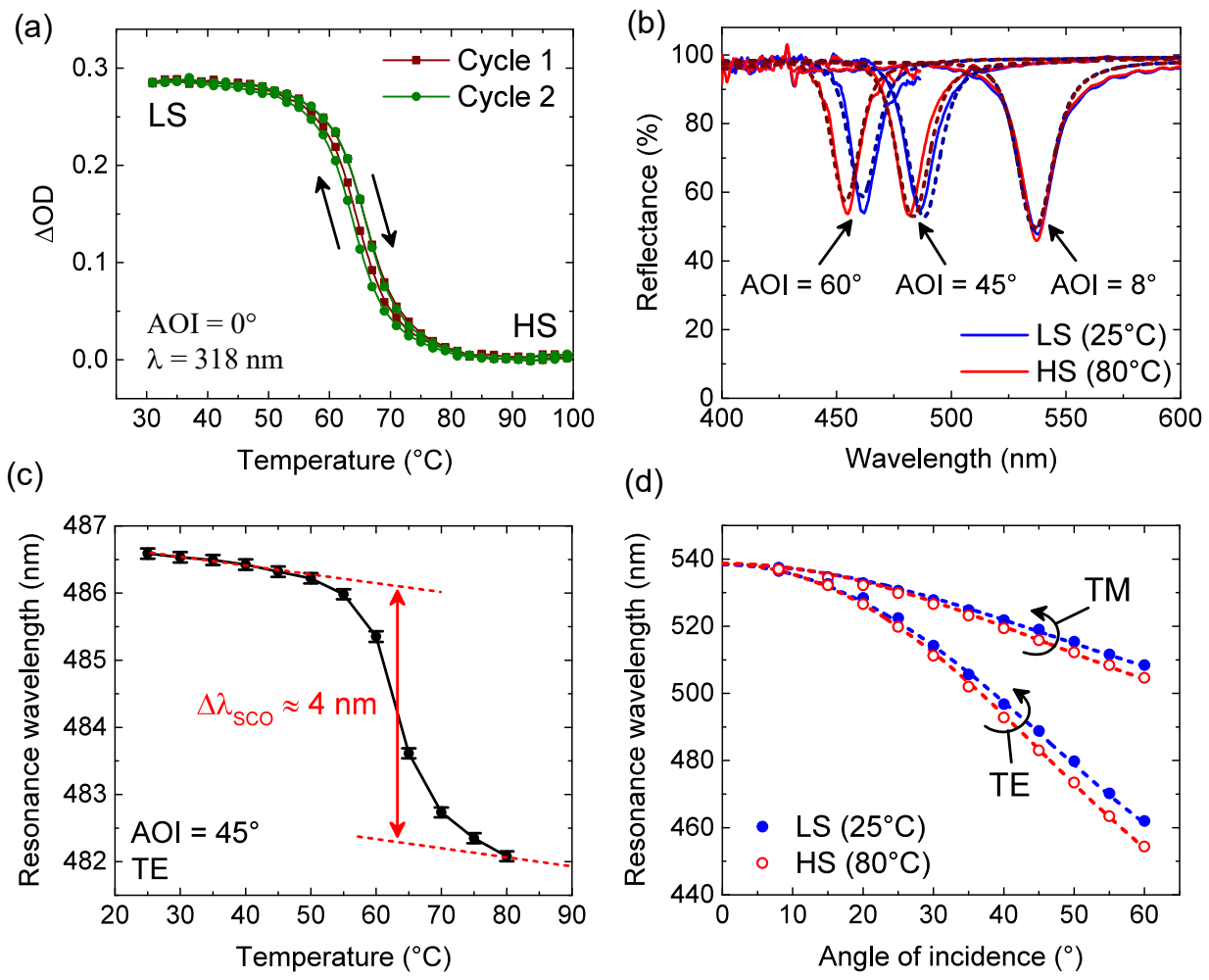

(d)

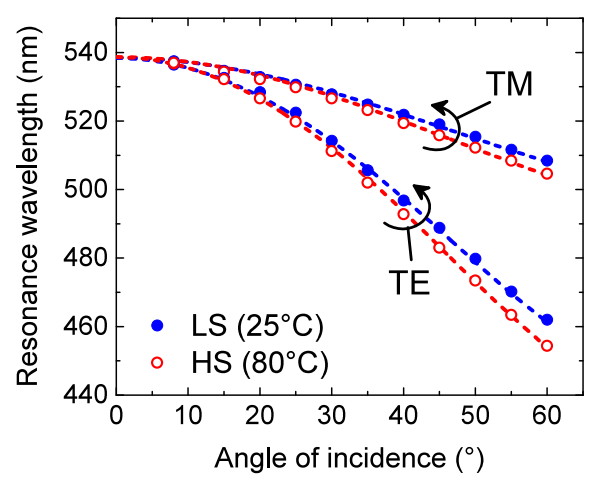

\section{Conclusions}

In summary, we have shown that thin films of the molecular SCO complex $\left[\mathrm{Fe}\left(\mathrm{HB}(1,2,4-\text { triazol-1-yl })_{3}\right)_{2}\right]$ exhibit a substantial change of their refractive index $(\Delta n=0.2-0.04)$ in the UV-Vis-NIR spectral ranges upon the spin transition, whereas absorption losses remain negligible $\left(k \leq 10^{-4}\right)$ for wavelengths above ca. $350 \mathrm{~nm}$. High-quality films of this compound were then incorporated into metal-dielectric-metal stacks by straightforward vacuum evaporation. The resulting Fabry-Perot cavities displayed spectrally 
tuneable resonances (up to $8 \mathrm{~nm}$ shift), which could be traced back - using transfer matrix calculations - to the refractive index and film thickness changes upon the near-room-temperature spin transition. The present results, together with the previously demonstrated large bandwidth and high cycling endurance of the films (in ambient conditions), demonstrate that the quality of spin crossover nanomaterials has reached today performance thresholds, which qualify them as viable technological solutions for demanding photonic applications. As such, this work opens up prospects for a wealth of reconfigurable and self-adaptive applications, including spatial light modulators, photonic integrated circuits and tuneable optical components - with particular relevance for the visible wavelength range wherein other families of PCMs fall short.

\section{Author contributions}

Device design and transfer matrix calculations (SC, HC), SCO film deposition and characterization (VS), ellipsometry (SP), synthesis and characterization of the SCO complex (LS, LR), device fabrication (YZ, IS), device characterization (YZ, KR, GM), project supervision $(A B, G M)$. All authors discussed the results and contributed to the preparation of the manuscript.

\section{Conflicts of interest}

There are no conflicts to declare.

\section{Acknowledgment}

This work was supported by a CNRS 'Emergence@INC' grant (LR), by a CNRS MITI 'Nouveaux Matériaux' grant (SC) as well as by the LAAS-CNRS micro and nanotechnologies platform, member of the French RENATECH network of cleanroom facilities. The PhD of Y. Z. was supported by a China Scholarship Council grant.

\section{Notes and references}

1 Z. Yang and S. Ramanathan, IEEE Photonics J., 2015, 7, 0700305.

2 K. J. Miller, R. F. Haglund and S. M. Weiss, Opt. Mater. Exp., 2018, 8, 2415.

3 J. Beeckman, K. Neyts and P. J. M. Vanbrabant, Opt. Eng., 2011, 50, 081202.

4 W. Dong, H. Liu, J. K. Behera, L. Lu, R. J. H. Ng, K. V. Sreekanth, X. Zhou, J. K. W. Yang and R. E. Simpson, Adv. Funct. Mater., 2019, 29, 1806181.

5 Yun Meng, J. K. Behera, Y. Ke, L. Chew, Y. Wang, Y. Long and R. E. Simpson, Appl. Phys. Lett., 2018, 113, 071901.

6 C. Williams, N. Hong, M. Julian, S. Borg and H. J. Kim, Opt. Exp., 2020, 28, 10583.

7 P. A. Do, A. Hendaoui, E. Mortazy, M. Chaker and A. Haché, Opt. Commun., 2013, 288, 23.

8 K. Makino, K. Kato, Y. Saito, P. Fons, A. V. Kolobov, J. Tominaga, T. Nakano and M. Nakajima, J. Mater. Chem. C, $2019,7,8209$.

9 J. D. Ryckman, V. Diez-Blanco, J. Nag, R. E. Marvel, B. K. Choi, R. F. Haglund and S. M. Weiss, Opt. Express, 2012, 20, 13215.

10 H. Khandelwal, E. P. A. van Heeswijk, A. P. H. J. Schenning and M. G. Debije, J. Mater. Chem. C, 2019, 7, 7395.

11 J. Faneca, T. D. Bucio, F. Y. Gardes and A. Baldycheva, Appl. Phys. Lett., 2020, 116, 093502.

12 G. Molnár, S. Rat, L. Salmon, W. Nicolazzi and A. Bousseksou, Adv. Mater., 2018, 30, 1703862.

13 P. Gütlich, A. Hauser and H. Spiering, Angew. Chem. Int. Ed. Engl. 1994, 33, 2024.

14 A. Hauser, Chem. Phys. Lett., 1993, 202, 173.

15 P. Mounaix, N. Lascoux, J. Degert, E. Freysz, A. Kobayashi, N. Daro and J.-F. Létard, Appl. Phys. Lett., 2005, 87, 244103.

16 P. Mounaix, E. Freysz, J. Degert, N. Daro, J.-F. Létard, P. Kužel, V. Vigneras and L. Oyenhart, Appl. Phys. Lett., $2006,89,174105$.

17 E. D. Loutete-Dangui, F. Varret, E. Codjovi, P. R. Dahoo, H. Tokoro, S. Ohkoshi, C. Eypert, J.-F. Létard, J. M. Coanga and K. Boukheddaden, Phys. Rev. B, 2007, 75, 184425.

18 C. Arnaud, T. Forestier, N. Daro, E. Freysz, J.-F. Létard, G. Pauliat and G. Roosen, Chem. Phys. Lett., 2009, 470, 131.

19 G. Félix, K. Abdul-Kader, T. Mahfoud, I. A. Gural'skiy, W. Nicolazzi, L. Salmon, G. Molnár and A. Bousseksou, J. Am. Chem. Soc., 2011, 133, 15342.

20 A. Akou, I. A. Gural'skiy, L. Salmon, C. Bartual-Murgui, C. Thibault, C. Vieu, G. Molnár and A. Bousseksou, J. Mater. Chem., 2012, 22, 3752.

21 I. A. Gural'skiy, C. Quintero, K. Abdul-Kader, M. Lopes, C. Bartual-Murgui, L. Salmon, G. Molnár, A. Bousseksou, P. Zhao and D. Astruc, J. Nanophotonics, 2012, 6, 063517.

22 K. Abdul-Kader, M. Lopes, C. Bartual-Murgui, O. Kraieva, E. M. Hernández, L. Salmon, W. Nicolazzi, F. Carcenac, C. Thibault, G. Molnár and A. Bousseksou, Nanoscale, 2013, 5, 5288.

23 A. Akou, C. Bartual-Murgui, K. Abdul-Kader, M. Lopes, G. Molnár, C. Thibault, C. Vieu, L. Salmon, and A. Bousseksou, Dalton Trans., 2013, 42, 16021.

24 A. lazzolino, G. Galle, J. Degert, J.-F. Létard and E. Freysz, Chem. Phys. Lett., 2015, 641, 14.

25 C. Bartual-Murgui, A. Akou, C. Thibault, G. Molnár, C. Vieu, L. Salmon and A. Bousseksou, J. Mater. Chem. C $2015,3,1277$.

26 M. Palluel, N. M. Tran, N. Daro, S. Buffière, S. Mornet, E. Freysz and G. Chastanet, Adv. Funct. Mater., 2020, 30, 2000447.

27 V. Shalabaeva, S. Rat, M. D. Manrique-Juarez, A.-C. Bas, L. Vendier, L. Salmon, G. Molnár and A. Bousseksou, J. Mater. Chem. C, 2017, 5, 4419. 
28 V. Shalabaeva, M. Mikolasek, M. D. Manrique-Juarez, A.-C. Bas, S. Rat, L. Salmon, W. Nicolazzi, G. Molnár and A. Bousseksou, J. Phys. Chem. C, 2017, 121, 25617.

29 V. Shalabaeva, A.-C. Bas, M. Piedrahita-Bello, K. Ridier, L. Salmon, C. Thibault, W. Nicolazzi, G. Molnár and A. Bousseksou, Small, 2019, 15, 1903892.

30 K. Ridier, A.-C. Bas, V. Shalabaeva, W. Nicolazzi, L. Salmon, G. Molnár, A. Bousseksou, M. Lorenc, R. Bertoni, E. Collet and H. Cailleau, Adv. Mater., 2019, 31, 1901361.

31 K. Ridier, A.-C. Bas, Y. Zhang, L. Routaboul, L. Salmon, G. Molnár, C. Bergaud and A. Bousseksou, submitted.

32 V. Shalabaeva, K. Ridier, S. Rat, M. D. Manrique-Juarez, L. Salmon, I. Séguy, A. Rotaru, G. Molnár, and A. Bousseksou, Appl. Phys. Lett., 2018, 112, 013301.

33 L. Poggini, M. Gonidec, J. H. Gonzalez-Estefan, G. Pecastaings, B. Gobaut and P. Rosa, 2018, Adv. Electron. Mater., 4, 1800204.

34 M. D. Manrique-Juarez, F. Mathieu, V. Shalabaeva, J. Cacheux, S. Rat, L. Nicu, T. Leïchlé, L. Salmon, G. Molnár and A. Bousseksou, Angew. Chem. Int. Ed., 2017, 56, 8074.

35 K. Ridier, Y. Zhang, M. Piedrahita-Bello, C. M. Quintero, L. Salmon, G. Molnár, C. Bergaud and A. Bousseksou, Adv. Mater. 2020, 32, 2000987.

36 S. H. Wemple and M. DiDomenico, Jr., Phys. Rev. Lett., 1969, 23, 1156.

37 S. Rat, K. Ridier, L. Vendier, G. Molnár, L. Salmon and A. Bousseksou, CrystEngComm, 2017, 19, 3271.

38 M. Born, and E. Wolf, Principles of Optics, Cambridge University Press, UK, 1999.

39 P. B. Johnson and R. W. Christy, Phys. Rev. B, 1972, 6, 4370. 